\title{
Reactivity worth measurements with CALIBAN and SILENE experimental reactors
}

\author{
P. Casoli ${ }^{\mathrm{a}}$ and N. Authier \\ CEA, Centre d'Études de Valduc, 21120 Is-sur-Tille, France
}

\begin{abstract}
Reactivity worth measurements of material samples put in the central cavities of nuclear reactors allow to test cross section nuclear databases or to extract information about the critical masses of fissile elements. Such experiments have already been completed on the CALIBAN and SiLENE experimental reactors operated by the Criticality and Neutronics Research Laboratory of Valduc (CEA, France), using the perturbation measurement technique. Calculations have been performed to prepare future experiments on new materials.
\end{abstract}

\section{Introduction}

Studies are currently carried out to improve the performances of neutron transport codes in order to increase the accuracy of simulations. To reach that goal, new data are needed to test the calculation codes. In that context, reactivity worth measurements are particularly interesting.

Such measurements also allow to study neutron properties of various materials and to test nuclear cross section databases.

The experiments presented in this paper are reactivity worth measurements based on the perturbation measurement technique. These experiments, carried out in experimental reactor central cavities, only need small amounts of materials.

\section{Characteristics of Valduc's experimental reactors}

\subsection{The facilities of the Criticality and Neutronics Research Laboratory of Valduc}

The Criticality and Neutronics Research Laboratory is located on the French Atomic Energy Commission (CEA) research center of Valduc.

It manages several experimental facilities:

- three experimental nuclear reactors [1],

- two electrostatic accelerators,

- a sub-critical apparatus,

- a bench devoted to the airborne release studies.

The three reactors are called Caliban, Prospero and Silene. Caliban and Prospero are two metallic core reactors, mainly composed of ${ }^{235} \mathrm{U}$. Silene is a liquid reactor, using a highly enriched ${ }^{235} \mathrm{U}$ nitrate solution.

The two electrostatic accelerators, named SAMES and Alvarez, produce mono-energetic neutron beams of $2.5 \mathrm{MeV}$ or $14 \mathrm{MeV}$ respectively by DD or DT fusion reactions.

The sub-critical facility, named Apparatus B, is an adjustable tool used to carry out reference experiments for qualifying criticality tools.

\footnotetext{
${ }^{a}$ Corresponding author, email address: pierre.casoli@cea.fr
}

The bench Bise allows to measure airborne release fraction of radioactive powders, deposits or pellets, used for safety analysis of nuclear facilities.

Furthermore the laboratory manages a large inventory of nuclear materials on various physicochemical forms: solutions ( $\mathrm{U}$ and $\mathrm{Pu}$ ), fuel rods $(\mathrm{U}$ and $\mathrm{Pu})$ and metals $(\mathrm{U})$.

\subsection{Configurations of the reactors}

Caliban is a metallic core cylindrical reactor. Its diameter is $19.5 \mathrm{~cm}$ and its height is $25 \mathrm{~cm}$. It is composed of two blocks: a fixed block and a mobile one. Each block is made of five metallic plates of a molybdenum and highly enriched uranium alloy. Four cylindrical control rods, made of the same alloy, allow to operate the reactor following two modes: a steady state power mode and a pulsed one.

Prospero is also a metallic core cylindrical reactor. Its diameter is $31.4 \mathrm{~cm}$ and its height is $35.2 \mathrm{~cm}$. Like Caliban, it is composed of a fixed block and a mobile one. Each block is made of two metallic plates of a highly enriched uranium alloy. The core is surrounded by a natural uranium and steel reflector, in which are placed four cylindrical natural uranium control rods, which allow to operate the reactor following a steady state power mode.

Unlike Caliban, whose axis is vertical, Prospero is horizontally oriented. The consequence is that the rod position measurements on Prospero are not accurate enough to allow perturbation measurements.

Silene, which means "Source d'Irradiation à Libre Évolution NEutronique - Irradiation Source following Neutron Free Evolution", is a liquid core reactor, whose fuel is a highly enriched ${ }^{235} \mathrm{U}$ nitrate solution, confined in a steel annular tank. The latter has a $36.8 \mathrm{~cm}$ external diameter and is $103.6 \mathrm{~cm}$ high. The reactor is managed by a control rod made of a neutron absorber material placed in the central channel. Three operating modes are possible: a steady state one, a pulsed one and a "free evolution" one.

Each reactor includes a central irradiation cavity, situated between the two blocks for CALIBAn and Prospero, and inside the control rod for SILENE. 


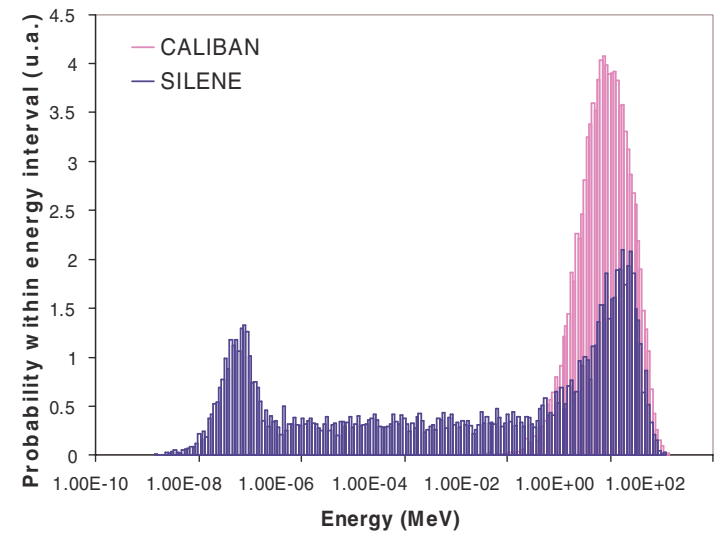

Fig. 1. Neutron spectra in Caliban and Silene irradiation cavities.

\subsection{Performances of Caliban and Silene reactors}

The main characteristics of CALIBAN and SILENE reactors in the steady state mode, which is the mode used for the perturbation technique, are given in table 1 .

Table 1. Performances of Caliban and SiLENE in steady state mode.

\begin{tabular}{lll}
\hline & CALIBAN & SILENE \\
\hline Maximum power (W) & 600 & 10000 \\
Maximum power (fissions/s) & $2 \times 10^{13}$ & $3.3 \times 10^{14}$ \\
Operation limitation (fissions) & $3.6 \times 10^{17}$ & $5 \times 10^{17}$ \\
Neutron fluence (for $10^{17}$ fissions) & $2.4 \times 10^{14}$ & $10^{14}$ \\
Neutron mean energy (MeV) & 1.4 & 0.8 \\
\hline
\end{tabular}

Neutron fluence and energy given in table 1 are values available in the reactor irradiation cavities and calculated by the TRIPOLI simulation code [2]. These calculations allow also to determine neutron spectra in the cavities, displayed on figure 1.

Table 2. Neutron spectra in Caliban and Silene irradiation cavities.

\begin{tabular}{lll}
\hline & CALiban & Silene \\
\hline Thermal neutrons $(\mathrm{E}<0.5 \mathrm{eV})$ & $0 \%$ & $23.5 \%$ \\
Epithermal neutrons $(0.5 \mathrm{eV}<\mathrm{E}<1 \mathrm{MeV})$ & $57.1 \%$ & $54.2 \%$ \\
Fast neutrons $(\mathrm{E}>1 \mathrm{MeV})$ & $42.9 \%$ & $22.3 \%$ \\
\hline
\end{tabular}

The rough subdivision of the spectra in three intervals displayed on table 2 as a first approach shows the different characteristics of the reactors. CALIBAN has a fission spectrum, while SiLENE spectrum has mainly epithermal and thermal components.

\section{Reactivity worth measurements}

\subsection{Principle}

Perturbation measurement experiments consist in inserting small sample of materials in the central cavity and in driving the reactor to the critical state. The comparison with the positions of the control rods leading to the critical state without any sample in the cavity allow to determine the reactivity worth of the material.

Experimental $\mathrm{k}_{\mathrm{eff}}$ coefficients and reactivity worth values can also be determined by another method: the reactor is driven to a slightly supercritical configuration and its large period is converted into reactivity through the Inhour equation.

\subsection{Experimental method}

A perturbation measurement experiment is composed of two steps:

- a critical approach of the reactor, without any sample in the cavity,

- a second approach with the material sample in the cavity, all other parameter staying equal to the first step.

In fact, the material sample is placed in a little steel hollow cylinder called "pawn", which is maintained in the cavity by a sample carrier. The void pawn and the sample carrier should be placed in the cavity for the first step approach.

The critical state is reached on CaLIBAN by the insertion of one of the control rod (BC3) and on SILENE by the adjustment of the liquid level in the tank. For both reactors, the difference of rod or liquid level between the two steps gives the reactivity worth of the sample.

To obtain the reactivity worth in standard units, reactors have to be calibrated. This work consists in determining the conversion factors from the rod or liquid level measured in length units into reactivity worth in pcm (or $\left.10^{-5}\right)$ units.

Calibrations can then be validated by perturbation method experiments on materials whose reactivity worth is already well known (e.g., $\left.{ }^{235} \mathrm{U}\right)$.

\subsection{Feasibility experiments}

Several experiments have already been carried out in the past on Caliban and Silene, which demonstrate the feasibility of the perturbation measurements on these reactors.

${ }^{235} \mathrm{U},{ }^{237} \mathrm{~Np}$ and ${ }^{239} \mathrm{Pu}$ samples have been designed and placed into CALIBAN central cavity for perturbation measurements. These experiments allowed to study the neutron properties of these materials and ${ }^{237} \mathrm{~Np}$ data were interpreted to discriminate nuclear databases. Furthermore a feasibility study about experiment on ${ }^{241} \mathrm{Am}$ has been completed.

A perturbation measurement completed on SILENE using a $\mathrm{CH}_{2}$ capsule allowed to determine the reactivity worth of this material. The liquid levels at the critical state with and without the $360 \mathrm{~g} \mathrm{CH}_{2}$ sample were respectively 307.05 and 311.31 $\mathrm{mm}$. The calibration of the reactor giving a conversion factor of $80 \mathrm{pcm} / \mathrm{mm}$, the reactivity worth of the material has been calculated as follow:

$$
\rho=(311.31-307.05) \times 80 / 360=1 \mathrm{pcm} / \mathrm{g} .
$$




\subsection{Accuracy of the measurements}

The accuracy of the reactivity worth values depends on the accuracy of the rod or liquid level measurements.

On Caliban, the position of the control rods are known with a precision of about $0.01 \mathrm{~mm}$ and the rough estimate of the conversion factor is about $10 \mathrm{pcm} / \mathrm{mm}$, so the accuracy of the reactivity worth values is about $0.1 \mathrm{pcm}$.

On SILENE, the liquid level is measured with a precision of about $0.01 \mathrm{~mm}$ and the rough estimate of the conversion factor is about $80 \mathrm{pcm} / \mathrm{mm}$, so the accuracy of the reactivity worth values is about $1 \mathrm{pcm}$.

\section{Simulations}

\subsection{Studied materials}

Until now, fissile actinides have been studied in Caliban and SILENE.

The aim of this study is to show that perturbation method experiments on CALIBAN and SiLENE are relevant for different types of materials: fissile or not fissile major and minor actinides, fission products, neutron reflectors or absorbers or other materials which are less sensitive to neutrons.

To determine the materials used in our calculations, absorption cross sections [3] of several isotopes have been examined and compared to CALIBAN and SiLENE spectra.

First calculations have also been performed with beryllium, copper, tantalum and neptunium 237.

\subsection{Spherical modeling}

Calculations have been carried out using the one dimensional version of the Panda code [4]. This neutron transport deterministic code, based on the $\mathrm{S}_{\mathrm{N}}$ method, was developed by the CEA for criticality and stochastic applications.

In a first step, critical radii of the Caliban and Silene fuels have been calculated, to determine the equivalent spheres of each reactor.

Then, reactivity coefficients of the systems, with or without samples in the cavities, have been determined by simulations. The thicknesses of the reactor steel walls are taken into account and the cylindrical cavities are modeled by same radius spheres.

\subsection{Results}

Calculations have been performed using the ENDF-B6 nuclear database cross sections and the MoReT code [5] 172 energy group distribution.

Results are displayed in tables 3 and 4.

In these calculations, sample masses have been chosen as big as possible, taking into account the material densities and the irradiation cavity volumes. The aim was to obtain important reactivity worth values, higher than the reactor accuracy values (respectively about 0.1 and $1 \mathrm{pcm}$ for CALIBAN and SiLENE).

Results show that the obtained values for most materials are clearly above the measurement limits. This demonstrates
Table 3. Results of the simulations on the CaLiban reactor.

\begin{tabular}{lccc}
\hline & & \multicolumn{2}{c}{ Reactivity worth } \\
& Mass $(\mathrm{g})$ & $(\mathrm{pcm})$ & $(\mathrm{pcm} / \mathrm{g})$ \\
\hline Beryllium & 13 & 54 & 4.15 \\
Copper & 60 & -3 & -0.05 \\
Tantalum & 110 & -27 & -0.25 \\
Neptunium 237 & 100 & 255 & 2.55 \\
\hline
\end{tabular}

Table 4. Results of the simulations on the SILENE reactor.

\begin{tabular}{lccc}
\hline & & \multicolumn{2}{c}{ Reactivity worth } \\
& Mass $(\mathrm{g})$ & $(\mathrm{pcm})$ & $(\mathrm{pcm} / \mathrm{g})$ \\
\hline Beryllium & 300 & 250 & 0.83 \\
Copper & 500 & -321 & -0.64 \\
Tantalum & 500 & -213 & -0.42 \\
\hline
\end{tabular}

the feasibility of the perturbation experiments with these materials.

Next simulations and experiments will be carried out with smaller size samples. The smaller the size of the sample, the less modified is the neutron flux in the reactor cavity. The measurement analyses will then be of better quality with small samples.

\subsection{Interpretations}

Calculations show the different reactivity worth values (in $\mathrm{pcm} / \mathrm{g}$ ) obtained for a material in CALIBAN or SiLENE, which point out the effect of the difference between the two reactor spectra and particularly the effect of the thermal and epithermal components of SiLENE spectrum. A benchmark of Caliban [6] has recently been achieved which will allow a more accurate knowledge of the neutron flux in the cavity and a better interpretation of our experiments.

The reactivity worth value of ${ }^{237} \mathrm{~Np}$ measured in CALIBAN spectra can be compared with the experimental data presented in ref. [7]. Three 16.32, 32.66 and $48.98 \mathrm{~g}$ mass ${ }^{237} \mathrm{~Np}$ samples have been successively placed in CALIBAN cavity. The reactivity worth measurements range from 1.589 to $1.649 \mathrm{pcm}$. Our calculated value is about $60 \%$ above these experimental results. This difference can be mainly explained by the approximations coming from the one dimensional model. Particularly, the simulated flux shape in a spherical geometry is different from the experimental flux shape in the real cylindrical geometry.

Simulations performed with the PANDA code two dimensional version are currently under progress, which will permit to model the systems more precisely. These new results will be compared with experimental values and will allow to discuss the performances of different nuclear cross section databases.

\section{Conclusion}

The Criticality and Neutronics Research Laboratory of Valduc is managing several facilities and a large inventory of nuclear materials devoted to neutron property studies. The two experimental reactors CALIBAN and SiLENE are adapted for reactivity worth measurements by the perturbation method. Such experiments allow to determine the neutrons properties 
of small size material sample placed in the irradiation central cavity of the reactors.

\section{References}

1. N. Authier et al., CEA Technical Note, SRNC 04-01, 2004 (unpublished).

2. J.-P. Both et al., CEA Technical Report, DEN DM2S/SERMA/ RT/ 01-2901/B (2001) (unpublished).
3. JANIS 2.1, NEA Data Bank, OCDE, 2004.

4. P. Humbert, in Proceedings of the ANS Math $\mathcal{E}$ Computation Meeting, Gatlinburg, 2003.

5. B. Normand et al., IRSN Technical Note, DPEA SEC/T/02.281 (2003) (unpublished).

6. N. Authier, B. Mechitoua, HEU-MET-FAST-080, NEA/NSC/ DOC(95)03/I, OCDE/NEA (to be published).

7. P. Humbert, B. Mechitoua, in Proceedings of the Seventh International Conference on Nuclear Criticality Safety, Part 1, Tokai (2003), p. 204. 